\title{
A numerical model of rotating bearings for thermo-mechanical coupled analysis
}

\author{
J. Nam, H. S. Ryou \& S. W. Cho \\ School of Mechanical Engineering, \\ Chung-Ang University, South Korea
}

\begin{abstract}
The function of the bearing is to support radial and axial forces and reduce the frictional force about rotating elements. Bearings are widely used in rail wheels and turbines having rotating elements like shafts. Heat is necessarily generated in bearings due to frictional forces between balls, inner and outer rings. Heat generation in bearings affects the thermal expansion of bearings and performance of lubrication. So, if the heat generated due to frictional force is not predicted properly, a failure of bearings might occur earlier time than expected. If they were broken when unexpected, it would damage the whole system. So, it is important to predict the heat generation in bearings accurately. Theoretical, experimental and numerical research on heat generation in bearings has been performed for a long time. It is difficult to predict temperature in bearings through experiment or theoretical analysis, because the highest temperature is in the local area and they are usually dependent on each shape of the bearings. Local heat generation in bearings accelerates the failure of bearings. In this study, we suggested the numerical model to predict heat generated by frictional force between contact surfaces and investigate thermal distribution in bearing systems by using the finite element method (FEM). The energy created by frictional force calculated in contact analysis is converted into heat energy. Most numerical research to predict distribution of temperature in bearings is considered in the steady-state disregarding the effect of rotation. However, it is important to consider the effect of rotation which affects the thermal distribution and lubrication. So, we used the thermo-mechanical coupled model to calculate heat generation, considering the effect of rotation.

Keywords: bearings, numerical simulation, thermal distribution, bearing load.
\end{abstract}




\section{Introduction}

Research has been performed to investigate the characteristics of bearings like frequency, allowable loads and others. Bearings are used in various mechanical parts in the industrial field. Recently, mechanical elements having higher performance like turbo engines or the wheels of high-speed trains have been developed. Bearings used in high-speed trains are exposed to high rpm and severe load boundary conditions.

Frictional heat is necessarily generated in bearings due to rolling friction. So, high performance bearings are required in high-speed trains where higher frictional heat is generated than in normal conditions. High temperature affects the performance of lubrication and also increases contact pressure due to thermal expansion. The higher contact pressures generate additional frictional heat, so it might fall into the positive feedback state. If frictional heat is not properly controlled or predicted, it would accelerate the decreasing life expectancy of bearings.

So it is important to identify the thermal characteristic and the temperature distribution of bearings. Various studies have been performed to predict temperature on bearings. Earlier studies were theoretical studies and suggested a mathematical model predicting the frictional heat of bearings for two dimensional models [1-7]. Research based on the mathematical method estimated the frictional heat energy by converting the poser loss of the shafts supported by bearings. But, the method has some limitations in that it could not predict three-dimensional situations and it can calculate only the global frictional heat on the bearing systems.

Recently, studies have been performed to predict three-dimensional frictional heat by using a high speed computer and by employing the finite element method (FEM) [8-14]. Some numerical studies were carried out by applying the heat flux as boundary condition and heat energy was converted from power loss by a mathematical model [8-10].

Above thermal analysis is known to match well in the global system, which includes bearing components [9]. But these methods do not consider the rotation of the bearing components, which is roller, inner and outer rings. For example, brake systems having a rotating element have a heat bend on the disc due to friction between a brake disc and a fixed pad. The rotational effect should be considered in order to accurately identify the temperature distribution in the bearings.

The coupled thermos-mechanical analysis uses a Lagrangian approach in the commercial FEM program. A Lagrangian approach is suitable for the coupled thermal and structural analysis having small displacements. If a problem had large deformation, it is too difficult to obtain the converged solution because of the contact algorithms and excessive computational times. So, previous studies did not consider the bearing rotations.

A numerical model that did not consider the rotation of bearings could predict the thermal distribution only in the global system, and it cannot predict temperature and thermal expansion on local components like roller, inner and outer ring. It is more sensitive in bearings with higher performance. 
In this study, we compared the effect of the rotation characteristics in the thermal distribution of the system. We also identified the limitations of the Lagrangian approach. The commercial finite element system ANSYS 16.0. has been employed for analysis of the study.

\section{Simulation}

\subsection{Numerical modelling}

Various types of bearings like ball, cylinder roller and taper roller have been used in the industry. The purpose of this study is to identify the effect of rotation for thermal distribution of bearings. This study as an initial step of the research is aimed to solve the temperature distribution of the rotating bearings. The first numerical model using the Lagrangian approach has some limitations, and another method is required for the analysis

We created a two-dimensional numerical bearing model with roller bearings. Our numerical bearing model consists of roller bearings, outer and inner rings. The actual bearing systems also have cages that help in uniformly locating the roller bearings. Since the cages can be considered by using the appropriate boundary conditions, they are ignored in the modelling which accelerated the simulation efficiency by reducing the contact areas [7].

In this study, we applied a constraint conditions that coupled the degree of freedom (rotation) about the origin on center node of roller bearings. The geometry information of bearing system, which are roller bearing, inner and outer rings, and the material properties are listed in tables 1 and 2.

Table 1: The geometry information of bearings.

\begin{tabular}{|c|c|}
\hline Geometry & Size $(\mathrm{mm})$ \\
\hline Inner diameter of IR & 40 \\
\hline Thickness of IR & 10 \\
\hline Inner diameter of OR & 90 \\
\hline Thickness of OR & 10 \\
\hline The number of bearings & 10 \\
\hline Diameter of roller & 15 \\
\hline
\end{tabular}

Table 2: The mechanical properties of the bearing system.

\begin{tabular}{|c|c|}
\hline Properties & Value \\
\hline Density & $7800 \mathrm{~kg} / \mathrm{m}^{3}$ \\
\hline Elastic modulus & $225 \mathrm{GPa}$ \\
\hline Poisson's ratio & 0.3 \\
\hline Coefficient of conduction & $50 \mathrm{~W} / \mathrm{m}-\mathrm{K}$ \\
\hline Specific heat & $470 \mathrm{~J} / \mathrm{Kg}-\mathrm{C}^{\circ}$ \\
\hline Coefficient of thermal expansion & $12 \times 10^{-6}$ \\
\hline
\end{tabular}


Figure 1 shows the numerical model. The numerical model has 35,658 elements and 10,224 nodes.
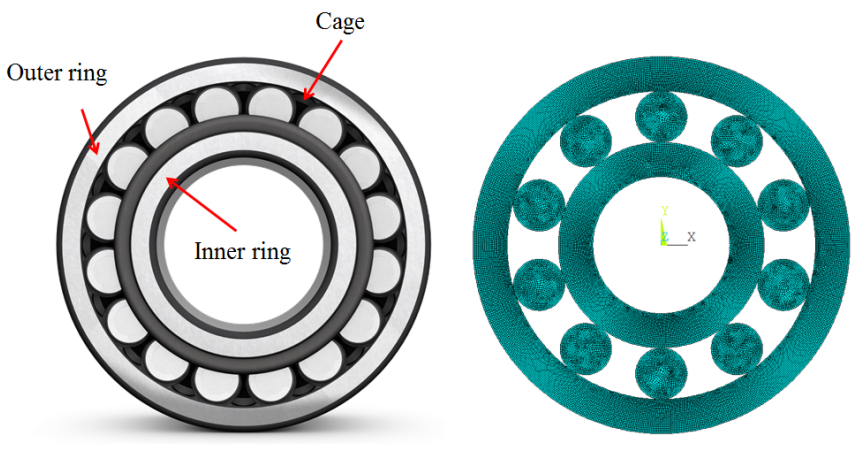

Figure 1: Numerical model of bearing system in 2D.

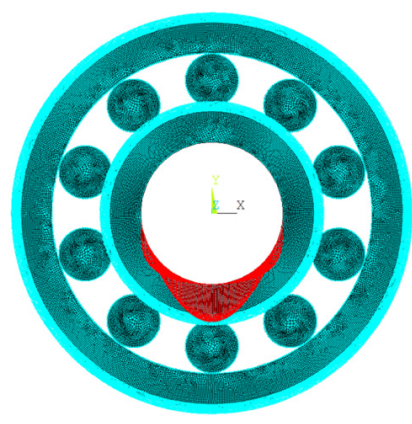

(a)

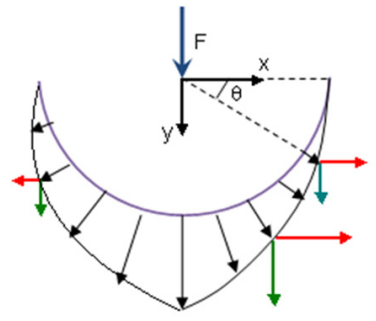

(b)

Figure 2: (a) Initial boundary condition; (b) parabolic distribution of bearing load.

\subsection{Numerical analysis}

ANSYS 16.0 has the capability of directly calculating the heat generation by using the thermal-structural element coupling technique. This method that calculates the heat generation through contact analysis was suggested by P. Wriggers in 1944. It is called thermo-mechanical analysis [11]. The equation calculating the heat energy is eqn (1) and it is applied as a heat source, where $V$ is the sliding rate, FHTG is the ratio from frictional dissipated energy converted into heat.

$$
q=F H T G \times \mu \times p \times V
$$


Contact analysis was divided two couple, between inside surface of outer bearings and roller bearings, between outside surface of inner ring and bearings. Augmented Lagrangian method and initial contact condition were applied in contact analysis. If contact analysis has many contact area, it is not easy to get the converged solution. So, we did not create cage of bearing, we applied the boundary condition that couple a degree of freedom into the tangential direction.

Bearings have many contact areas due to the applied loads in rotational, vertical direction and pre-stress due to the manufacturing tolerances. So it was taken long time to solve contact problem. If pre-stress is not applied, roller bearings would not rotate. Loads were applied to a step by step manner in order to apply the above mentioned three types of loads

Figure 2(a) shows initial boundary conditions. First the inside of the inner ring was fixed for tangential direction. Vertical load which is the bearing load was applied to the inner ring, and pre-pressure for radial direction. In this study, types of bearing load are in parabolic distribution. Bearing loads are given in eqns (2)(4).

$$
\begin{gathered}
F_{x}=F_{o} \times(\cos \theta) \times(\sin \theta)^{2} \\
F_{y}=F_{o} \times(\sin \theta) \times(\sin \theta)^{2} \\
F=\sum_{i=0}^{n}\left(F_{o}\right)_{i} \sin ^{3} \theta
\end{gathered}
$$

\subsection{Numerical results}

Figure 3 shows the contour of contact pressure and frictional stresses on contact surface between bearings and rings when first load step ended. Bottom roller bearings have the largest contact pressure due to bearing load. Figure 4 shows the contour of the temperatures. Figure 4(a) did not consider the rotational effect. It had uniform contour of temperature. Figure 4(b) considered the rotations of the outer ring. When components of bearing system are rotating, contact point would be relocated beside previous location like neutral point in rolling mill. During the short time interval, temperatures in the bearing systems were shown in fig. 4(b).

Heat conduction occurs in a time-dependent manner. Heat could not be sufficiently conducted during short time interval. The reason is that the area having temperature information was small. Additionally, because the value of temperature and thermal distribution are so sensitive to FHTG, it is important to select FHTG. It is too difficult to calculate the coefficient of FHTG which is the rate that heat was converted from frictional forces. In real situations, FHTG is smaller than 1E-8 and is also affected by geometry of the bearings. If we could calculate the FHTG accurately and have high performance computer to solve a complicated problem of contact analysis, it is possible to identify the thermal distribution of rotating bearings. 


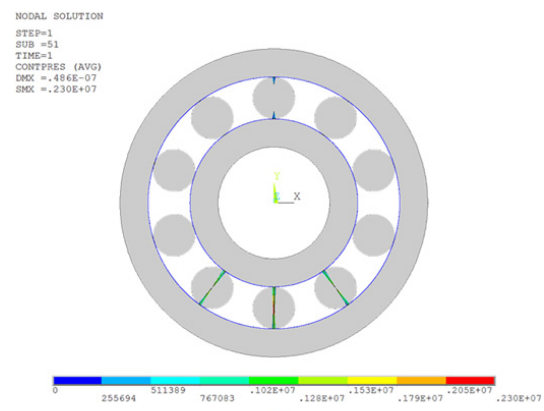

(a)

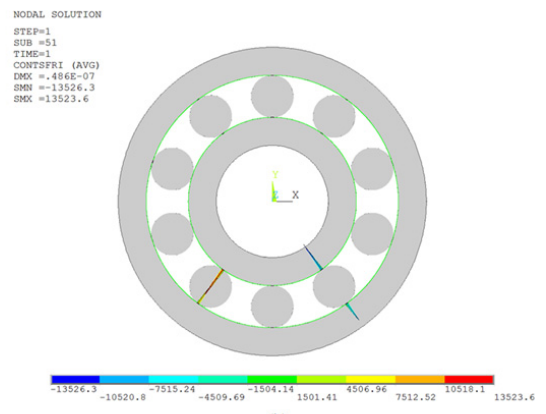

(b)

Figure 3: Contour of (a) contact pressure; (b) frictional force on contact surface.

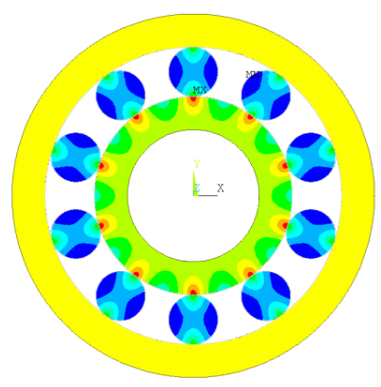

(a)

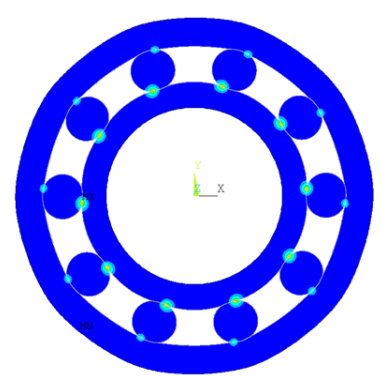

(b)

Figure 4: Temperature contour (a) steady-state (b) rotating state.

\section{Conclusion}

In this study, we performed research on the rotational effects that are related to thermal distribution in the rotating elements. The effect of the rotation in the analysis considered is performed for comparison. So, when identifying the local temperature distribution in the bearing system, you should consider the rotational effects.

But the Lagrangian approach for the analysis of the solid domain is not proper in the large deformation situation like rotating elements. And a problem having many contact areas like a bearing system is usually time consuming. It is hard to get the converged solution due to complicated boundary condition and many contact areas.

Even when the heat flux boundary condition is the same, the temperature distribution is not the same because heat conduction is a time-dependent phenomena in nature. For the temperatures to reach the steady-state, it requires considerably long time during which the bearings have rotated a substantial amount of cycles while heat energy was transferring. So, it is too difficult to 
perfectly solve the problem about rotating bearings using only a Lagrangian approach. In future work, we have planned to apply a Eulerian approach used in the fluid domain on a rotating bearing system in order to predict thermal distribution.

\section{Acknowledgement}

This research was supported by the Fire Fighting Safety and 119 Rescue Technology Research and Development Program funded by the Ministry of Public Safety and Security (MPSS-Fire Fighting Safety-2012-64).

\section{References}

[1] Kleckner, R. J., Pirvics, J. \& Castelli, V., High Speed Cylindrical Rolling Element Bearing Analysis - "CYBEAN" - Analytic Formulation. Journal of Lubrication Technology, 102(3), pp. 380-388, 1980.

[2] Zhang, Z., Qiu, X. \& Hong, Y., EHL analysis of rib-roller end contact in tapered roller bearing. Tribol Trans, 31(4), pp. 461-467, 1988.

[3] Nelias, D., Bercea, I. \& Mitu, N., Analysis of double-row tapered roller bearings, Part II - Results: prediction of fatigue life and heat dissipation. Tribol Transa, 46(2), pp. 240-247, 2003.

[4] Ai, S., Wang, W., Wang, Y. \& Zhao, Z., Temperature rise of double-row tapered roller bearings analyzed with the thermal network method. Tribology International, 87, pp. 11-22, 2015.

[5] Suh, J. \& Palazzolo, A., Three-Dimensional Thermohydrodynamic Morton Effect Simulation - Part I: Theoretical Model. Journal of Tribology, 136(3), 031706, 2014.

[6] Jin, C., Wu, B. \& Hu, Y., Heat generation modeling of ball bearing based on internal load distribution. Tribology International, 45(1), pp. 8-15, 2012.

[7] Edwin, L. J., Numerical model to study of contact force in a cylindrical roller bearing with technical mechanical event simulation. Journal of Mechanical Engineering and Automation, 1(1), pp. 1-7, 2011.

[8] Houpert, L., Ball bearing and tapered roller bearing torque: analytical. numerical and experimental results. Tribol trans, 45(3), pp. 345-353, 2002.

[9] Kim, K. S., Lee, D. W., Lee, S. M., Lee, S. J. \& Hwang, J. H., A numerical approach to determine the frictional torque and temperature of an angular contact ball bearing in a spindle system. International Journal of Precision Engineering and Manufacturing, 16(1), pp. 135-142, 2015.

[10] Pouly, F., Changenet, C., Ville, F., Velex, P. \& Damiens, B., Power loss predictions in high-speed rolling element bearings using thermal networks. Tribol Trans, 53(6), pp. 957-967, 2010.

[11] Wriggers, P. \& Miehe, C., Contact constraints within coupled thermomechanical analysis a finite element model. Computer Methods in Applied Mechanics and Engineering, 113(3-4), pp. 301-319, 1994. 